\title{
Random User Activity with Mixed Delay Traffic
}

\author{
Homa Nikbakht and Michèle Wigger \\ LTCI, Télécom Paris, Institut Polytechnique de Paris \\ \{homa.nikbakht, michele.wigger\}@ telecom-paris.fr
}

\author{
Shlomo Shamai (Shitz) \\ Technion \\ sshlomo@ee.technion.ac.il
}

\begin{abstract}
This paper analyses the multiplexing gain (MG) achievable over a general interference network with random user activity and random arrival of mixed-delay traffic. The mixeddelay traffic is composed of delay-tolerant traffic and delaysensitive traffic where only the former can benefit from receiver cooperation since the latter is subject to stringent decoding delays. Two setups are considered. In the first setup, each active transmitter always has delay-tolerant data to send and delaysensitive data arrival is random. In the second setup, both delaytolerant and delay-sensitive data arrivals are random, and only one of them is present at any given transmitter. The MG regions of both setups are completely characterized for Wyner's softhandoff network. For Wyner's symmetric linear and hexagonal networks inner bounds on the MG region are presented.
\end{abstract}

\section{INTRODUCTION}

This paper presents coding schemes for the transmission of heterogeneous traffic with delay-sensitive and delay-tolerant data over interference networks with random user activity and random data arrivals. Delay-sensitive data, called "fast" messages, are subject to stringent delay constraints and their encoding and decoding processes cannot be delayed. Delaytolerant data, called "slow" messages, are subject to softer delay constraints and can benefit from receivers cooperation. Such mixed-delay constraints have been studied in [1]-[6].

This paper further takes into account random activities of the users. Specifically, in each transmission block only a subset of the users has a message to convey to its corresponding Rxs. The impact of random user activity on cellular networks has been previously studied in [7]-[9].

In this work, we combine random user activity with such mixed-delay constraints. We specifically consider two setups. In both setups, receivers (Rxs) can cooperate to decode their desired "slow" messages but not to decode "fast" messages. Each transmitter (Tx) is active with probability $\rho \in[0,1]$, and the goal is to maximize the average expected "slow" rate of the network, while the rate of each "fast" message is fixed to a target value. In the first setup, each active Tx transmits a "slow" message, and with probability $\rho_{f} \in[0,1]$ also transmits an additional "fast" message. In the second setup, each active Tx sends either a "fast" message with probability $\rho_{f}$ or a "slow" message with probability $1-\rho_{f}$.

For both setups, we propose general coding schemes and characterize their achievable multipleing gain (MG) regions for three networks: Wyner's soft-handoff network, Wyner's symmetric network and the hexagonal network. The achievable MG region is shown to be optimal for Wyner's soft-handoff network. In both setups, the obtained MG regions show that the average "slow" MG decreases i) with increasing number of interfering links, and ii) with increasing activity parameter $\rho$. The obtained MG regions also show that in the first setup, the maximum sum-MG is always attained at 0 "fast" MG, and increasing the "fast" MG decreases the sum-MG by a penalty that roughly speaking increases with the number of interference links in the network and with the activity parameter $\rho$. In contrast, in the second setup, for certain parameters the sum-MG is achieved at maximum "fast" MG and thus increasing the "fast" MG provides a gain in sum-MG, where we observe that the gain decreases with the number of interferers and the activity parameter $\rho$.

\section{RANDOM "FAST" ARRIVALS ONLY}

Consider a cellular network with $K$ Tx-Rx pairs $k=$ $1, \ldots, K$. Each Tx $k \in \mathcal{K} \triangleq\{1, \ldots, K\}$ is active with probability $\rho \in[0,1]$, in which case it sends a so called "slow" message $M_{k}^{(S)}$ to its corresponding Rx $k$. Here, $M_{k}^{(S)}$ is uniformly distributed over $\mathcal{M}_{k}^{(S)} \triangleq\left\{1, \ldots,\left\lfloor 2^{n R_{k}^{(S)}}\right\rfloor\right\}$, with $n$ denoting the blocklength and $R_{k}^{(S)}$ the rate of message $M_{k}^{(S)}$. Given that Tx $k$ is active, with probability $\rho_{f} \in[0,1]$, it also sends an additional "fast" message $M_{k}^{(F)}$ to Rx $k$. These "fast" messages are subject to stringent delay constraints, as we describe shortly, and uniformly distributed over the set $\mathcal{M}^{(F)} \triangleq\left\{1, \ldots,\left\lfloor 2^{n R^{(F)}}\right\rfloor\right\}$. "Fast" messages are thus all of same size and same rate $R^{(F)}$.

We introduce the i.i.d Bernoulli- $\rho$ random variables $A_{1}, \ldots, A_{K}$ and the i.i.d Bernoulli- $\rho_{f}$ random variables $B_{1}, \ldots, B_{K}$ and define the active Tx-set as

$$
\mathcal{T}_{\text {active }} \triangleq\left\{k \in \mathcal{K}: A_{k}=1\right\},
$$

and the "fast" Tx-set as

$$
\mathcal{T}_{\text {fast }} \triangleq\left\{k \in \mathcal{K}: A_{k} \cdot B_{k}=1\right\} .
$$

Then, for each $k \in \mathcal{K}$, Tx $k$ computes its channel inputs $X_{k}^{n} \triangleq$ $\left(X_{k, 1}, \ldots, X_{k, n}\right) \in \mathbb{R}^{n}$ as

$$
X_{k}^{n}= \begin{cases}f_{k}^{(B)}\left(M_{k}^{(F)}, M_{k}^{(S)}\right), & \text { if } k \in \mathcal{T}_{\text {fast }} \\ f_{k}^{(S)}\left(M_{k}^{(S)}\right), & \text { if } k \in \mathcal{T}_{\text {active }} \backslash \mathcal{T}_{\text {fast }} \\ 0, & \text { if } \mathcal{T}_{\text {active }}^{c} .\end{cases}
$$

for some encoding functions $f_{k}^{(B)}$ and $f_{k}^{(S)}$ on appropriate domains satisfying the average block-power constraint

$$
\frac{1}{n} \sum_{t=1}^{n} X_{k, t}^{2} \leq \mathrm{P}, \quad \forall k \in \mathcal{K}, \quad \text { almost surely. }
$$


To describe the interference network, let $X_{k}^{n}$ denote Tx $k$ 's input signal and $Y_{k}^{n} \triangleq\left(Y_{k, 1}, \ldots, Y_{k, n}\right)$ Rx $k$ 's output signal and define the interference sets

$$
\begin{aligned}
& \mathcal{I}_{\mathrm{Rx}, k} \triangleq\left\{\tilde{k} \in \mathcal{K} \backslash\{k\}: X_{k}^{n} \text { interferes } Y_{\tilde{k}}^{n}\right\}, \\
& \mathcal{I}_{\mathrm{Tx}, k} \triangleq\left\{\tilde{k} \in \mathcal{K} \backslash\{k\}: X_{\tilde{k}}^{n} \text { interferes } Y_{k}^{n}\right\} .
\end{aligned}
$$

The input-output relation of the network is then described as

$$
Y_{k, t}=A_{k} X_{k, t}+\sum_{\tilde{k} \in \mathcal{I}_{\mathrm{T} x, k}} A_{\tilde{k}} h_{\tilde{k}, k} X_{\tilde{k}, t}+Z_{k, t}
$$

where $\left\{Z_{k, t}\right\}$ are independent and identically distributed (i.i.d.) standard Gaussians for all $k$ and $t$ and independent of all messages; $h_{\tilde{k}, k}>0$ is the channel coefficient between Tx $\tilde{k}$ and $\mathrm{Rx} k$ and is a fixed real number smaller than 1 ; and $X_{0, t}=0$ for all $t$.

Each Rx $k \in \mathcal{T}_{\text {fast }}$ decodes the "fast" message $M_{k}^{(F)}$ based on its own channel outputs $Y_{k}^{n}$. So, it produces:

$$
\hat{M}_{k}^{(F)}=g_{k}^{(n)}\left(Y_{k}^{n}\right),
$$

for some decoding function $g_{k}^{(n)}$ on appropriate domains. It is assumed that receivers can fully cooperate on all receive signals when decoding their "slow" messages. So,

$$
\hat{M}_{k}^{(S)}=c_{k}^{(n)}\left(Y_{1}^{n}, \ldots, Y_{K}^{n}\right),
$$

where $c_{k}^{(n)}$ is a decoding function on appropriate domains.

Given $\mathrm{P}>0$, a pair $\left(R^{(F)}(\mathrm{P}), \bar{R}^{(S)}(\mathrm{P})\right)$ is said achievable, if for each $K>0$, there exist rates $\left\{R_{k}^{(S)}\right\}_{k=1}^{K}$ satisfying

$$
\bar{R}^{(S)} \leq \varlimsup_{K \rightarrow \infty} \frac{1}{K} \mathbb{E}\left[\sum_{k \in \mathcal{T}_{\text {active }}} R_{k}^{(S)}\right],
$$

and encoding, cooperation, and decoding functions satisfying constraint (4) and so that the probability of error

$$
\mathbb{P}\left[\bigcup_{k \in \mathcal{T}_{\text {fast }}}\left(\hat{M}_{k}^{(F)} \neq M_{k}^{(F)}\right) \text { or } \bigcup_{k \in \mathcal{T}_{\text {active }}}\left(\hat{M}_{k}^{(S)} \neq M_{k}^{(S)}\right)\right]
$$

goes to 0 as $n \rightarrow \infty$. An MG pair $\left(\mathrm{S}^{(F)}, \mathrm{S}^{(S)}\right)$ is called achievable, if for all powers $\mathrm{P}>0$ there exist achievable average rates $\left\{R^{(F)}(\mathrm{P}), \bar{R}^{(S)}(\mathrm{P})\right\}_{\mathrm{P}>0}$ satisfying

$$
\begin{aligned}
& \mathrm{S}^{(F)} \triangleq \varlimsup_{\mathrm{P} \rightarrow \infty} \frac{R^{(F)}(\mathrm{P})}{\frac{1}{2} \log (\mathrm{P})} \cdot \rho \rho_{f}, \\
& \mathrm{~S}^{(S)} \triangleq \varlimsup_{\mathrm{P} \rightarrow \infty} \mathbb{E}\left[\frac{\bar{R}^{(S)}(\mathrm{P})}{\frac{1}{2} \log (\mathrm{P})}\right] .
\end{aligned}
$$

The closure of the set of all achievable MG pairs $\left(\mathrm{S}^{(F)}, \mathrm{S}^{(S)}\right)$ is called fundamental $M G$ region and is denoted $\mathcal{S}^{\star}\left(\rho, \rho_{f}\right)$.

The MG in (11) measures the average expected "slow" MG on the network. Since the "fast" rate is fixed to $R^{(F)}$ at all Txs in $\mathcal{T}_{\text {fast }}$, we multiply the MG in (10) by $\rho \rho_{f}$ to obtain the average expected "fast" MG of the network.

\section{A. Achievable MG Region and Coding Schemes}

In this section, we propose two schemes, one with large "fast" MG and the other with zero "fast" MG.
1) Transmitting at large $S^{(F)}$ : Since we wish to transmit at maximum "fast" MG, each "fast" transmission should not be interfered (except for signals up to noise level) by any other ("fast" or "slow") transmission. Therefore, we partition $\mathcal{K}$ into $\delta$ subsets $\mathcal{K}_{1}, \ldots, \mathcal{K}_{\delta}$, for some positive integer $\delta$, in a way that all the signals sent by Txs in a given subset $\mathcal{K}_{i}$ do not interfere, i.e., for each $i \in\{1, \ldots, \delta\}$ :

$$
k^{\prime} \notin \mathcal{I}_{\mathrm{Rx}, k^{\prime \prime}} \quad \text { and } \quad k^{\prime \prime} \notin \mathcal{I}_{\mathrm{Rx}, k^{\prime}}, \quad \forall k^{\prime}, k^{\prime \prime} \in \mathcal{K}_{i} .
$$

We divide the total transmission time into $\delta$ equally-sized phases. In the $i$-th phase,

- each Tx $k$ in $\mathcal{K}_{i} \cap \mathcal{T}_{\text {fast }}$ sends its entire "fast" message $M_{k}^{(F)}$ but no part of the "slow" message $M_{k}^{(S)}$;

- each Tx $k \in \mathcal{K} \backslash\left(\mathcal{K}_{i} \cap \mathcal{T}_{\text {fast }}\right)$ sends a part of its "slow" message $M_{k}^{(S)}$ if

$$
\mathcal{I}_{\mathrm{Rx}, k} \cap \mathcal{T}_{\text {fast }} \cap \mathcal{K}_{i}=\emptyset ;
$$

otherwise it does not send anything.

Condition (13) ensures that transmissions of "fast" messages are not interfered at all. By (12), the condition is in particular satisfied for all $k \in \mathcal{K}_{i} \cap\left(\mathcal{T}_{\text {active }} \backslash \mathcal{T}_{\text {fast }}\right)$.

The described scheme achieves a "fast" rate of $R^{(F)}=$ $\frac{1}{\delta} \frac{1}{2} \log (1+\mathrm{P})$, and thus by (10), a "fast" MG of

$$
\mathrm{S}_{\max }^{(F)}=\frac{\rho \rho_{f}}{\delta} \text {. }
$$

It also achieves an expected "slow" MG of

$\overline{\mathrm{S}}_{\text {coop }}^{(S)}(K)$

$$
\begin{aligned}
& =\frac{1}{K} \sum_{i=1}^{\delta} \frac{1}{\delta}\left(\sum_{k \in \mathcal{K}_{i}} \mathbb{P}\left\{k \in \mathcal{T}_{\text {active }} \backslash \mathcal{T}_{\text {fast }}\right\}\right. \\
& \left.\quad+\sum_{k \in \mathcal{K} \backslash \mathcal{K}_{i}} \mathbb{P}\left\{k \in \mathcal{T}_{\text {active }}\right\} \mathbb{P}\left\{\mathcal{I}_{\mathrm{Rx}, k} \cap \mathcal{T}_{\text {fast }} \cap \mathcal{K}_{i}=\emptyset\right\}\right) \\
& \left.=\frac{\rho}{\delta K} \sum_{i=1}^{\delta}\left(\left(1-\rho_{f}\right)\left|\mathcal{K}_{i}\right|+\sum_{k \in \mathcal{K} \backslash \mathcal{K}_{i}}\left(1-\rho \rho_{f}\right)^{\left|\mathcal{I}_{\mathrm{Rx}, k} \cap \mathcal{K}_{i}\right|}\right\}\right) .
\end{aligned}
$$

2) Transmitting at $\mathrm{S}^{(F)}=0$ : Each $\mathrm{Tx} k \in \mathcal{T}_{\text {active }}$ sends only a "slow" message but no "fast" message. Since perfect cooperation is assumed at the Rxs, each of the "slow" messages can be transmitted with MG 1 . The average expected "slow" MG over the network is therefore

$$
\overline{\mathrm{S}}_{\max }^{(S)}=\rho,
$$

while $\mathrm{S}^{(F)}=0$.

Time sharing the two schemes establishes the following:

Proposition 1 (Achievable MG Region): The inner bound on $\mathcal{S}^{\star}\left(\rho, \rho_{f}\right)$ contains the region:

convex hull $\left((0,0),\left(0, \overline{\mathrm{S}}_{\max }^{(S)}\right),\left(\mathrm{S}_{\max }^{(F)}, \overline{\mathrm{S}}_{\text {coop }}^{(S)}\right),\left(\mathrm{S}_{\max }^{(F)}, 0\right)\right)$.

In the following three sections we specialize this proposition to different interference networks. As we will see, for our first network, we can also prove a corresponding converse result, and thus Proposition 1 exactly characterizes $\mathcal{S}^{\star}\left(\rho, \rho_{f}\right)$. 


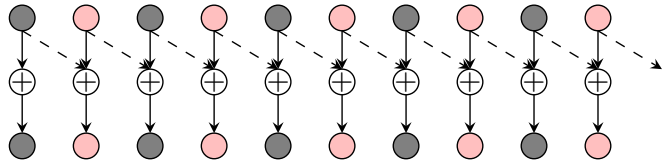

Fig. 1. An illustration of Wyner's soft-handoff network where the Tx/Rx pairs in $\mathcal{K}_{1}$ are colored in gray and the $\mathrm{Tx} / \mathrm{Rx}$ pairs in $\mathcal{K}_{2}$ in pink. The interference graph is depicted by black dashed lines.

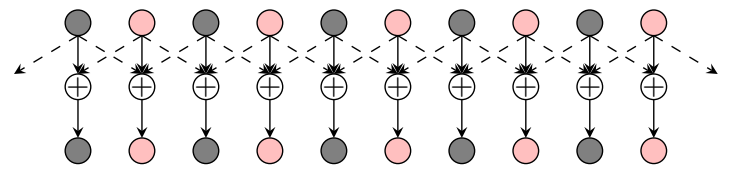

Fig. 2. An illustration of Wyner's symmetric network where the Tx/Rx pairs in $\mathcal{K}_{1}$ are colored in gray and the Tx/Rx pairs in $\mathcal{K}_{2}$ in pink. The interference graph is depicted by black dashed lines.

\section{B. Wyner's soft-handoff network}

Consider Wyner's soft-handoff network shown in Figure 1. Interference is short-range in the sense that the signal sent by $\mathrm{Tx} k$ is observed only by $\mathrm{Rx} k$ and by the neighbouring $\mathrm{Rx} k+1$. Thus $\mathcal{I}_{\mathrm{Rx}, k}=\{k+1\}$. For this network, we can exactly characterize the fundamental MG region $\mathcal{S}^{\star}\left(\rho, \rho_{f}\right)$ :

Theorem 1: The fundamental $\mathrm{MG}$ region $\mathcal{S}^{\star}\left(\rho, \rho_{f}\right)$ of Wyner's soft-handoff network is the set of all nonnegative pairs $\left(\mathrm{S}^{(F)}, \mathrm{S}^{(S)}\right)$ satisfying

$$
\begin{aligned}
\mathrm{S}^{(F)} & \leq \frac{\rho \rho_{f}}{2}, \\
(1+\rho) \mathrm{S}^{(F)}+\mathrm{S}^{(S)} & \leq \rho .
\end{aligned}
$$

Proof: The achievability part follows by specializing Proposition 1 to $\delta=2$ and to

$$
\mathcal{K}_{1}=\{1,3, \ldots, K-1\} \quad \text { and } \quad \mathcal{K}_{2}=\{2,4, \ldots, K\} .
$$

For this choice $\left|\mathcal{I}_{\mathrm{Rx}, k} \cap \mathcal{K}_{i}\right|=1$. The proof of the converse bound (20) is straightforward. The converse bound (21) is proved in Appendix A.

By above theorem, the fundamental $\mathrm{MG}$ region $\mathcal{S}^{\star}\left(\rho, \rho_{f}\right)$ is a quadrilateral, see also Fig. 4 , and it is mostly determined by the activity parameter $\rho$; the "fast" arrival probability $\rho_{f}$ only determines the vertical maximum-S $S^{(F)}=\frac{\rho \rho_{f}}{2}$ boundary of the region. The maximum-S ${ }^{(S)}$ boundary, which characterizes the maximum achievable "slow" $\mathrm{MG} \mathrm{S}^{(S)}$ in function of the "fast" $\mathrm{MG} \mathrm{S}^{(F)}$, is fully characterized by $\rho$ : it's a line segment with slope $-(1+\rho)$. In general, this slope determines the penalty that the maximum "slow" $\mathrm{MG} \mathrm{S}^{(S)}$ incurrs when one increases the "fast" MG. This penalty increases with increasing $\rho$ because with more active Txs in the network the probability increases that a given active "fast" Tx is interfered by other active transmitters, which then have to be forced to send at "slow" MG 0 in order not to harm the achievable "fast" MG.

\section{Wyner's symmetric network}

Consider Wyner's symmetric network in Figure 2, where the signal sent by Tx $k$ is observed by Rxs $k$ and $k+1$, and also by $\operatorname{Rx} k-1$. Thus $\mathcal{I}_{\mathrm{Rx}, k}=\{k-1, k+1\}$ for each $k \in \mathcal{K}$.

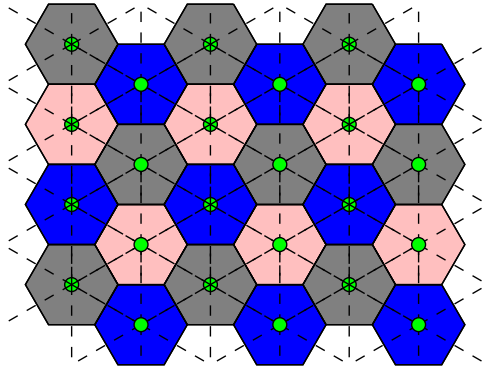

Fig. 3. An illustration of the hexagonal network where the $\mathrm{Tx} / \mathrm{Rx}$ pairs in $\mathcal{K}_{1}$ are colored in gray, the Tx/Rx pairs in $\mathcal{K}_{2}$ in blue and the Tx/Rxs in $\mathcal{K}_{3}$ in pink. The interference graph is depicted by black dashed lines.

Corollary 1: The fundamental $\mathrm{MG}$ region $\mathcal{S}^{\star}\left(\rho, \rho_{f}\right)$ of Wyner's symmetric model includes all nonnegative pairs $\left(\mathrm{S}^{(F)}, \mathrm{S}^{(S)}\right)$ satisfying

$$
\begin{aligned}
\mathrm{S}^{(F)} & \leq \frac{\rho \rho_{f}}{2}, \\
\left(1+\rho\left(2-\rho \rho_{f}\right)\right) \mathrm{S}^{(F)}+\mathrm{S}^{(S)} & \leq \rho .
\end{aligned}
$$

Proof: Specialize Proposition 1 to $\delta=2$ and to the sets $\mathcal{K}_{1}$ and $\mathcal{K}_{2}$ in (22). For this choice $\left|\mathcal{I}_{\mathrm{Rx}, k} \cap \mathcal{K}_{i}\right|=2$.

The region in above corollary is again a quadrilateral, but the maximum- $S^{(S)}$ boundary is now determined by both parameters $\rho$ and $\rho_{f}$ as its slope is $-\left(1+\rho\left(2-\rho \rho_{f}\right)\right)$. The dependency on $\rho_{f}$ however vanishes as $\rho \cdot \rho_{f} \rightarrow 0$ in which case the slope approaches $-(1+2 \rho)$. Interestingly, this asymptotic slope shows a factor 2 compared to the slope of the maximum- $\mathrm{S}^{(S)}$ boundary in Wyner's soft-handoff network. The reason is that in Wyner's symmetric network $\left|\mathcal{I}_{\mathrm{Tx}, k}\right|=2$ whereas in Wyner's soft-handoff network $\left|\mathcal{I}_{\mathrm{Tx}, k}\right|=1$. In the next subsection, we will see that in the hexagonal network where $\left|\mathcal{I}_{\mathrm{Tx}, k}\right|=6$, this asymptotic slope is $-(1+6 \rho)$.

\section{Hexagonal network}

Consider the hexagonal network in Figure 3 with $K$ hexagonal cells and each cell including one Tx and one Rx. The signals of $\mathrm{Tx} / \mathrm{Rx}$ pairs that lie in a given cell interfere with the signals sent in the 6 adjacent cells. The interference pattern is depicted by the dashed black lines in Fig. 3.

Corollary 2: The multiplexing gain region $\mathcal{S}^{\star}\left(\rho, \rho_{f}\right)$ includes all nonnegative pairs $\left(\mathrm{S}^{(F)}, \mathrm{S}^{(S)}\right)$ satisfying

$$
\begin{aligned}
\mathrm{S}^{(F)} & \leq \frac{\rho \rho_{f}}{3}, \\
\left(1+2 \rho\left(3-3 \rho \rho_{f}+\rho^{2} \rho_{f}^{2}\right)\right) \mathrm{S}^{(F)}+\mathrm{S}^{(S)} & \leq \rho .
\end{aligned}
$$

Proof: Follows by specializing Proposition 1 to $\delta=3$ and to appropriate sets $\mathcal{K}_{1}, \mathcal{K}_{2}$ and $\mathcal{K}_{3}$ shown in Fig. 3. Figure 4 evaluates the regions in Theorem 1 and in Corollaries 1 and 2 for $\rho=0.8$ and $\rho_{f}$ either 0.3 or 0.6 . We observe the quadrilateral shapes of all three regions.

\section{RANDOM "FAST" AND "SLOW" ARRIVALS}

The setup considered in this section differs from the previous setup only in that Txs in $\mathcal{T}_{\text {fast }}$ only send a "fast" message but no "slow" message. Thus, defining

$$
\mathcal{T}_{\text {slow }} \triangleq\left\{k \in \mathcal{K}: A_{k} \cdot\left(1-B_{k}\right)=1\right\},
$$




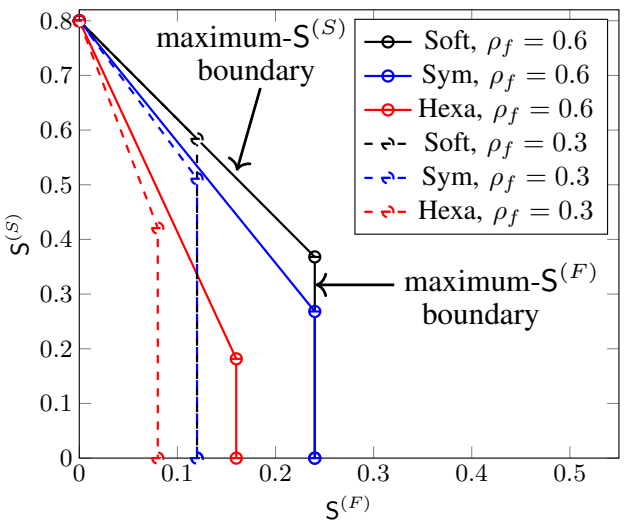

Fig. 4. MG Region $\mathcal{S}^{\star}\left(\rho, \rho_{f}\right)$ of different networks with $\rho=0.8$ and different values of $\rho_{f}$.

we have

$$
X_{k}^{n}= \begin{cases}f_{k}^{(F)}\left(M_{k}^{(F)}\right), & \text { if } k \in \mathcal{T}_{\text {fast }} \\ f_{k}^{(S)}\left(M_{k}^{(S)}\right), & \text { if } k \in \mathcal{T}_{\text {slow }} \\ \mathbf{0} & \text { if } k \in \mathcal{T}_{\text {active }}^{c}\end{cases}
$$

for some function $f_{k}^{(F)}$ and $f_{k}^{(S)}$ on appropriate domains that satisfy the average block-power constraint (4). All other definitions are as in the previous Section II. We denote the fundamental MG region for this setup by $\mathcal{S}_{2}^{*}\left(\rho, \rho_{f}\right)$.

\section{A. Achievable MG Region and Coding Schemes}

We again propose two schemes, one for large "fast" MG and the other for zero "fast" MG.

1) Transmitting at large $\mathrm{S}^{(F)}$ : Similar to the scheme presented in Subsection II-A1, we partition $\mathcal{K}$ into sets $\mathcal{K}_{1}, \ldots, \mathcal{K}_{\delta}$ and divide the total transmission time into $\delta$ equally-sized phases. In the $i$-th phase, each $\operatorname{Tx} k$ in $\mathcal{K}_{i} \cap \mathcal{T}_{\text {fast }}$ sends its "fast" message and each Tx $k \in \mathcal{T}_{\text {slow }}$ sends its "slow" message if $\mathcal{I}_{\mathrm{Rx}, k} \cap \mathcal{T}_{\text {fast }} \cap \mathcal{K}_{i}=\emptyset$; otherwise it does not send any message. The described scheme achieves a "fast" MG of $\mathrm{S}_{\max }^{(F)}=\frac{\rho \rho_{f}}{\delta}$, and an expected "slow" $\mathrm{MG}$ of

$$
\begin{aligned}
& \overline{\mathrm{S}}_{\text {coop }, 2}^{(S)}(K) \\
& =\frac{1}{K} \sum_{i=1}^{\delta} \frac{1}{\delta}\left(\sum_{k \in \mathcal{K}_{i}} \mathbb{P}\left\{k \in \mathcal{T}_{\text {slow }}\right\}\right. \\
& \left.\quad+\sum_{k \in \mathcal{K} \backslash \mathcal{K}_{i}} \mathbb{P}\left\{k \in \mathcal{T}_{\text {slow }}\right\} \mathbb{P}\left\{\mathcal{I}_{\text {Rx }, k} \cap \mathcal{T}_{\text {fast }} \cap \mathcal{K}_{i}=\emptyset\right\}\right) \\
& \left.=\frac{\rho\left(1-\rho_{f}\right)}{\delta K} \sum_{i=1}^{\delta}\left(\left|\mathcal{K}_{i}\right|+\sum_{k \in \mathcal{K} \backslash \mathcal{K}_{i}}\left(1-\rho \rho_{f}\right)^{\left|\mathcal{I}_{\mathrm{Rx}, k} \cap \mathcal{K}_{i}\right|}\right\}\right) .
\end{aligned}
$$

2) Transmitting at $\mathrm{S}^{(F)}=0$ : Each $\mathrm{Tx} k \in \mathcal{T}_{\text {slow }}$ sends a "slow" message with MG 1 . The average expected "slow" MG over the network is therefore

$$
\overline{\mathrm{S}}_{\max , 2}^{(S)}=\rho\left(1-\rho_{f}\right) .
$$

Each Tx $k \in \mathcal{T}_{\text {fast }}$ remains silent and thus $\mathrm{S}^{(F)}=0$.
3) General Achievable $M G$ Region:

Proposition 2: The set $\mathcal{S}_{2}^{\star}\left(\rho, \rho_{f}\right)$ contains the region:

convex hull $\left((0,0),\left(0, \overline{\mathbf{S}}_{\max , 2}^{(S)}\right),\left(\mathrm{S}_{\max }^{(F)}, \overline{\mathrm{S}}_{\mathrm{coop}, 2}^{(S)}\right),\left(\mathrm{S}_{\max }^{(F)}, 0\right)\right)$.

We specialize this result to the interference networks introduced in Sections II-B, II-C and II-D using the same choices for $\delta$ and the sets $\left\{\mathcal{K}_{i}\right\}_{i=1}^{\delta}$. For Wyner's soft-handoff network this inner bound is again tight.

4) Wyner's Soft-Handoff Network:

Theorem 2: The fundamental MG region $\mathcal{S}_{2}^{\star}\left(\rho, \rho_{f}\right)$ is the set of all nonnegative pairs $\left(\mathrm{S}^{(F)}, \mathrm{S}^{(S)}\right)$ satisfying

$$
\begin{aligned}
\mathrm{S}^{(F)} & \leq \frac{\rho \rho_{f}}{2}, \\
\rho\left(1-\rho_{f}\right) \mathrm{S}^{(F)}+\mathrm{S}^{(S)} & \leq \rho\left(1-\rho_{f}\right) .
\end{aligned}
$$

Proof: Achievability follows by specializing Proposition 2 to $\delta=2$ and to the sets $\mathcal{K}_{1}$ and $\mathcal{K}_{2}$ in (22). For this choice $\left|\mathcal{I}_{\mathrm{Rx}, k} \cap \mathcal{K}_{i}\right|=1$. The proof of the converse is omitted.

Like in the previous setup, the fundamental MG region $\mathcal{S}_{2}^{\star}\left(\rho, \rho_{f}\right)$ is a quadrilateral. Interestingly, now all boundaries depend on both activity parameters $\rho$ and $\rho_{f}$, in particular the maximum "slow" MG equals $\rho\left(1-\rho_{f}\right)$. Moreover, the maximum sum-rate is not achieved for this maximum "slow" MG anymore. Formally, this holds because the slope of the maximum- $S^{(S)}$ boundary is $-\rho\left(1-\rho_{f}\right)$ and thus larger than -1 . So, the maximum sum-rate point is obtained for maximum "fast" $\mathrm{MG} \mathrm{S}^{(F)}=\frac{\rho \rho_{f}}{2}$. The underlying intuition is that for $\rho\left(1-\rho_{f}\right)<1$ it may occur that a "fast" MG can be accommodated without the need to sacrifice a "slow" MG when the single interferer is not active anyways.

Figure 5 illustrates $\mathcal{S}_{2}^{\star}\left(\rho, \rho_{f}\right)$ for Wyner's soft-handoff network as well as the inner bounds we obtain for Wyner's symmetric network and the hexagonal network under activity parameters $\rho=0.8$ and $\rho_{f}$ is either 0.3 or 0.6 .

5) Wyner's Symmetric Network:

Corollary 3: The $\mathrm{MG}$ region $\mathcal{S}_{2}^{\star}\left(\rho, \rho_{f}\right)$ includes all nonnegative pairs $\left(\mathrm{S}^{(F)}, \mathrm{S}^{(S)}\right)$ satisfying

$$
\begin{aligned}
\mathrm{S}^{(F)} & \leq \frac{\rho \rho_{f}}{2}, \\
\rho\left(1-\rho_{f}\right)\left(2-\rho \rho_{f}\right) \mathrm{S}^{(F)}+\mathrm{S}^{(S)} & \leq \rho\left(1-\rho_{f}\right) .
\end{aligned}
$$

Proof: Specialize Proposition 2 to $\delta=2$ and to $\mathcal{K}_{1}$ and $\mathcal{K}_{2}$ as in (22). For this choice $\left|\mathcal{I}_{\mathrm{Rx}, k} \cap \mathcal{K}_{i}\right|=2$.

Here the slope of the maximum- $S^{(S)}$ boundary is $-\rho(1-$ $\left.\rho_{f}\right)\left(2-\rho \rho_{f}\right)$ and can be larger or smaller than -1 depending on the activity parameters. So, depending on these parameters, the maximum sum-MG is either achieved for zero "fast" $\mathrm{MG}$ or for maximum "fast" MG. Typically, for large values of $\rho_{f}$, i.e., when most of the "active" Txs send "fast" messages, the maximum sum-MG is achieved at maximum "fast" MG. When $\rho_{f}$ is small and $\rho$ sufficiently large, then most of the users are active and intend to send "slow" messages. In this case, scheduling "fast" messages most likely comes at the expense of silencing active neighbours that wishing to send "slow" 


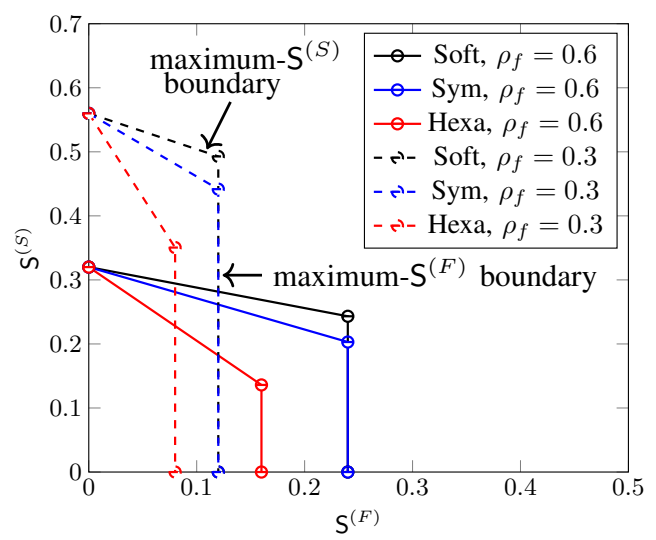

Fig. 5. Fundamental MG Region $\mathcal{S}_{2}^{\star}\left(\rho, \rho_{f}\right)$ of the three networks with $\rho=$ 0.8 and $\rho_{f}$ equal to 0.3 or 0.6 .

messages. It is further interesting to notice that in the limiting regime $\rho \rho_{f} \rightarrow 0$, the slope of the maximum- $S^{(S)}$ boundary approaches $-2 \rho\left(1-\rho_{f}\right)$ and is thus 2 times the slope in Wyner's soft-handoff network. As we will see, the hexagonal model treated next shows a factor 6 . For all three networks, the asymptotic slope in the limit $\rho \rho_{f} \rightarrow 0$ is thus given by $-\left|\mathcal{I}_{\mathrm{Tx}, k}\right| \rho\left(1-\rho_{f}\right)$.

6) The Hexagonal Model:

Corollary 4: The MG region $\mathcal{S}_{2}^{\star}\left(\rho, \rho_{f}\right)$ includes all nonnegative pairs $\left(\mathrm{S}^{(F)}, \mathrm{S}^{(S)}\right)$ satisfying

$$
\begin{aligned}
\mathrm{S}^{(F)} & \leq \frac{\rho \rho_{f}}{3}, \\
2 \rho\left(1-\rho_{f}\right)\left(3-3 \rho \rho_{f}+\rho^{2} \rho_{f}^{2}\right) \mathrm{S}^{(F)}+\mathrm{S}^{(S)} & \leq \rho\left(1-\rho_{f}\right) .
\end{aligned}
$$

\section{CONClusions}

We considered two different setups to simultaneously transmit delay-sensitive and delay-tolerant traffic over interference networks with randomly activated users. Under both setups, we characterized the multiplexing gain region of Wyner's softhandoff network and derived an inner bound on the MG region of any general interference network.

Our results show that in the first setup, where each active Tx always has "slow" (delay-tolerant) data to send, the sumMG is decreased with increasing "fast" (delay-sensitive) MG. The corresponding penalty mostly depends on the activity parameter $\rho$ and the interference set size $\left|\mathcal{I}_{\mathrm{Tx}, k}\right|$ of the network. It increases with both parameters, intuitively because more Txs have to be silent when accommodating "fast" transmissions.

In contrast, in the second setup where each active Tx has either a "slow" or a "fast" message to send, depending on the values of the activity parameters $\rho$ and $\rho_{f}$, the sum-MG is either achieved at maximum "fast" MG or at 0 "fast" MG. The former holds for small values of $\rho$ where only few Txs in the network are active and thus "fast" transmissions often can be accommodated without silencing active "slow" Txs.

An interesting line of future work consideres buffers to store not yet transmitted "slow" messages similar to [11].

\section{ACKNOWLEDGEMENT}

The work of $\mathrm{H}$. Nikbakth and M. Wigger was supported by the ERC under grant agreement no. 715111. The work of S.
Shamai has been supported by the European Union's Horizon 2020 Research And Innovation Programme, grant agreement no. 694630 .

\section{APPENDIX A}

\section{PROOF OF THE CONVERSE PART TO THEOREM 1}

Fix $K$ and realizations of the sets $\mathcal{T}_{\text {active }}$ and $\mathcal{T}_{\text {fast }}$. Following the steps in $\left[4\right.$, Section V], we prove that for each $k \in \mathcal{T}_{\text {active }}$ :

$$
\begin{aligned}
R_{k}^{(F)}+ & R_{k}^{(S)}+R_{k+1}^{(F)} \\
\leq & \frac{1}{2} \log \left(1+\left(1+\left|h_{k, k+1}\right|^{2}\right) \mathrm{P}\right)+\frac{1}{2} \log \left(1+\left|h_{k, k+1}\right|^{2}\right) \\
& \quad+\max \left\{-\log \left|h_{k, k+1}\right|, 0\right\}+\frac{\epsilon_{n}}{n},
\end{aligned}
$$

where $R_{k+1}^{(F)}$ is the rate of the "fast" message at Rx $k+1$, which is either 0 or equal to $R^{(F)}$. For simplicity, we abbreviate the RHS of (39) by $\Delta$, and we sum up this bound for all values of $k \in \mathcal{T}_{\text {active }}$ :

$$
\sum_{k \in \mathcal{T}_{\text {active }}}\left(R_{k}^{(F)}+R_{k}^{(S)}+R_{k+1}^{(F)}\right) \leq\left|\mathcal{T}_{\text {active }}\right| \cdot \Delta .
$$

Taking expectation over (39) and dividing by $K$, we obtain:

$$
\mathbb{E}\left[\bar{R}^{(S)}\right]+R^{(F)}\left(\rho \rho_{f}+\rho^{2} \rho_{f}\right) \leq \rho \cdot \Delta,
$$

because the expected number of indices $k \in \mathcal{T}_{\text {active }}$ for which $R_{k}^{(F)}=R^{(F)}$ equals $\rho \cdot \rho_{f}$ and the expected numbers of indices $k \in \mathcal{T}_{\text {active }}$ for which $R_{k+1}^{(F)}=R^{(F)}$ equals $\rho^{2} \cdot \rho_{f}$. Dividing by $\frac{1}{2} \log \mathrm{P}$ and letting $\mathrm{P} \rightarrow \infty$ proves (21).

\section{REFERENCES}

[1] K. M. Cohen, A. Steiner, and S. Shamai (Shitz) "The broadcast approach under mixed delay constraints," in Proc. IEEE ISIT 2012, Cambridge (MA), USA, July 1-6, pp. 209-213, 2012.

[2] R. Zhang, J. Cioffi, and Y.-C. Liang, "MIMO broadcasting with delayconstrained and no-delay-constrained services," in Proc. IEEE ICC 2005 , Seoul, South Korea, May 16-20, pp. 783-787, 2005.

[3] R. Zhang, "Optimal dynamic resource allocation for multi-antenna broadcasting with heterogeneous delay-constrained traffic," IEEE J. of Sel. Topics in Signal Proc., vol. 2, no. 2, pp. 243-255, Apr. 2008.

[4] H. Nikbakht, M. Wigger and S. Shamai (Shitz), "Mixed delay constraints in Wyners soft-handoff network," in Proc. IEEE ISIT, Vail, CO, USA, 17-22 June, pp. $1171-1175,2018$.

[5] A. Matera, R. Kassab, O. Simeone and U. Spagnolini, "Non-orthogonal eMBB-URLLC radio access for cloud radio access networks with analog fronthauling," Entropy, vol. 20, no. 9, pp. 661, 2018.

[6] H. Nikbakht, M. Wigger, W. Hachem and S. Shamai (Shitz), “ Mixed delay constraints on a fading C-RAN uplink," in Proc. IEEE ITW 2019, Visby, Sweden, Aug 25-28, 2019.

[7] O. Somekh, O. Simeone, H. V. Poor and S. Shamai (Shitz), "The two-tap input-earasure Gaussian channel and its application to cellular communications," in Proc. Allerton Conference on Communication, Control, and Computing, IL, USA, Sep 23-26, 2008.

[8] N. Levy and S. Shamai (Shitz), "Information theoretic aspects of users' activity in a Wyner-like cellular model," IEEE Trans. Inf. Theory, vol 56, pp. 2241-2248, Apr. 2010.

[9] O. Somekh, O. Simeone, H. V. Poor and S. Shamai (Shitz), "Throughput of cellular uplink with dynamic user activity and cooperative basestations," in Proc. IEEE ITW 2019, Taormina, Italy , Oct 11-16, 2009.

[10] A. D. Wyner, "Shannon-theoretic approach to a Gaussian cellular multiple-access channel," IEEE Trans. Inf. Theory, vol. 40, no. 6, pp. 1713-1727, Nov. 1994.

[11] A. Steiner and S. Shamai (Shitz), "On queuing and multilayer coding," IEEE Trans. Inf. Theory, vol. 56, no 5, pp. 2392-2415, 2010. 